\title{
ANÁLISIS COMPARATIVO ENTRE LOS INCENDIOS FORESTALES EN MONTE ALEGRE, BRASIL Y PINAR DEL RÍO, CUBA
}

\author{
Marcos Pedro Ramos Rodríguez ${ }^{1}$ \\ Ronaldo Viana Soares ${ }^{2}$
}

\begin{abstract}
RESUMEN
Los incendios forestales son hoy día un problema global. No obstante, en todos los casos, surgen y se desarrollan mostrando determinadas regularidades impuestas en lo fundamental por las condiciones meteorológicas, el combustible, las causas de su origen y la topografía. Comprender estas regularidades ayuda a entender el fenómeno para planificar su manejo. En este trabajo se hace un análisis comparativo del comportamiento histórico de los incendios forestales ocurridos de 1998 al 2001 en el Distrito Forestal de Monte Alegre, Estado de Paraná, sur de Brasil y en las Empresas Forestales de la Provincia de Pinar del Río, Cuba. Se ha considerado la distribución temporal - años, meses, horas, días de la semana - y espacial - grupos de especies, clases de bosque - de los incendios. También se analizaron las causas y algunos indicadores que muestran la eficiencia del servicio de protección. Se han obtenido resultados similares en ambos lugares, excepto para la época de incendios.
\end{abstract}

Palabras Claves: Comportamiento histórico, época de incendios, eficiencia

\section{COMPARATIVE ANALYSIS AMONG THE FOREST FIRES IN MONTE ALEGRE, BRASIL Y PINAR DEL RÍO, CUBA}

\begin{abstract}
Nowadays the forest fires are a global problem. Nevertheless, in all cases, they arise and they are developed showing certain regularities imposed in the fundamental thing by the meteorological conditions, the fuel, the causes of their origin and the topography. To understand these regularities help to get the phenomenon to planning their management. In this work a comparative analysis of the fire history is made since 1998 to 2001 in the Forest District of Monte Alegre, State of Paraná, south of Brazil and in the Forest Companies of the province of Pinar del Río, Cuba. It has been considered the temporary distribution - years, months, hours, days of week and space - groups of species, forest classes - of the fires. Also there have been analysed the causes and some indicators that show the efficiency of the protection service. Similar results have been obtained in both places, except for the fire season.
\end{abstract}

Keywords: Fire history, fire season, efficiency

\section{INTRODUCCIÓN}

Diferentes razones han provocado una considerable reducción de la superficie total cubierta de bosques. También han aumentado las actividades del hombre en los mismos y con estas, el peligro potencial de surgimiento de incendios, fenómeno que ha estado presente desde tiempos inmemoriales en distintas zonas del planeta, pero que hoy constituye una importante preocupación. Ellos reducen a cenizas a los ecosistemas forestales, insustituibles por ejemplo, como captadores de $\mathrm{CO}_{2}$. Además, el fuego envía a la atmósfera una gran cantidad de sustancias, algunas de las cuales contribuyen directamente al efecto invernadero. Según Calabri (1991) los incendios afectan a nivel mundial más de 10 millones de hectáreas de montes y otras superficies boscosas. Esto representa el 0,2 ó el 0,3 por ciento de la superficie total cubierta de vegetación boscosa. Hoy la tendencia tanto del número de incendios como de las superficies por ellos afectadas es al aumento. Esto ocurre a pesar de que por mucho tiempo se han obtenido resultados científico - técnicos que permiten pronosticarlo, prevenirlo y combatirlo cada vez con mayor eficiencia. Uno de estos resultados consiste en la evaluación del comportamiento

\footnotetext{
${ }^{1}$ Profesor, Ingeniero Forestal. Universidad de Pinar del Río, Cuba. Apartado Postal 268, Pinar del Río 1, Código 20100, Cuba. E-mail: cmramos@af.upr.edu.cu

${ }^{2}$ Profesor, Ingeniero Forestal. Universidade Federal do Paraná. Brasil. Avenida Lothario Meissner, 3400, Curitiba, PR, 80210-170 - Brasil. E-mail: rvsoares@floresta.ufpr.br
} 
histórico de los incendios en diferentes partes del mundo (Soares, 1988 y 1992, en Brasil; Vélez, 1990, en España; Canakcioglu, 1990, en Turquía; Ramos, 1999, en Pinar del Río, Cuba; etc.). Estas evaluaciones han permitido establecer que en todos los casos, surgen y se desarrollan mostrando determinadas regularidades impuestas en lo fundamental por las condiciones meteorológicas, el combustible, las causas de su origen y la topografía. Comprender estas regularidades ayuda a entender el fenómeno para planificar su manejo. Se ha visto que resulta muy importante comparar los resultados obtenidos en diferentes regiones. De esta forma se pueden intercambiar experiencias para obtener mejores resultados. También es posible planificar la cooperación entre las naciones tanto para acciones de lucha como para el desarrollo de programas de capacitación.

En este trabajo se hace un análisis comparativo del comportamiento histórico de los incendios forestales ocurridos de 1998 al 2001 en el Distrito Forestal de Monte Alegre, Estado de Paraná, sur de Brasil y en las Empresas Forestales de la Provincia de Pinar del Río, Cuba. Se ha considerado la distribución temporal - años, meses, horas, días de la semana - y espacial - grupos de especies, clases de bosque - de los incendios. También se analizaron las causas y la distribución de los incendios según las clases de tamaño, indicador que muestra la eficiencia del servicio de protección. Se han obtenido resultados similares en ambos lugares, excepto para la época de incendios.

\section{MATERIALES Y MÉTODOS}

El trabajo se desarrolló en las dos regiones siguientes:

- El Distrito Forestal de Monte Alegre, Brasil, el cual tiene una superficie total de $2000 \mathrm{~km}^{2}$, situado entre los $24^{\circ} 03^{\prime}$ y $24^{\circ} 31^{\prime}$ de latitud sur y entre los $50^{\circ} 10^{\circ}$ y $50^{\circ} 50^{\prime}$ de longitud oeste. La precipitación anual de la región es de 1399,5 mm, la temperatura media anual de $19^{\circ} \mathrm{C}$ y la humedad relativa del aire de 76,5 \% como media anual. Según Maack (1968) citado por Soares et al. (1974) de acuerdo con la clasificación de Köppen el clima de la región pertenece al tipo $\mathrm{Cfb}$ (Clima Templado con precipitaciones uniformes todo el año $\mathrm{y}$ verano caluroso pero con 4 ó más meses con temperatura media mensual del mes más caliente superior a $10^{\circ} \mathrm{C}$ ).

- La provincia de Pinar del Río, Cuba, con una superficie de $10901 \mathrm{~km}^{2}$, situada entre los $21^{\circ} 45^{\prime}$ de latitud norte y entre los $82^{\circ} 51^{\prime} y$ $84^{\circ} 57^{\circ}$ de longitud oeste. La precipitación anual de la región es de $1484,53 \mathrm{~mm}$, la temperatura media anual de $24,53^{\circ} \mathrm{C}$ y la humedad relativa del aire de $81 \%$ como media anual. Según Köppen (1936) citado por Wadsworth (2000) el clima es Aw (Clima Tropical con mes más frío con temperatura mayor de $18^{\circ} \mathrm{C}$ y una estación seca de al menos un mes con menos de $600 \mathrm{~mm}$ ).

Los datos fueron obtenidos para el caso de Monte Alegre en los archivos de la Sección de Defensa Contra Fuegos de la Industria Klabin do Paraná de Celulosa S/A. En el caso de Pinar del Río se obtuvieron en la oficina del Cuerpo de Guardabosques de esa región de protección. En ambos casos fueron procesados con ayuda del Sistema Integrado para el Manejo de Bases de Datos sobre Incendios Forestales (SIMBDIF) versión 1.2 (Ramos, 2002) y el Microsoft Excel.

El análisis siguió algunos de los elementos metodológicos desarrollados por Ramos (1999). En el caso de la distribución temporal de los incendios se tuvieron en cuenta años, meses, horas y días de la semana y en el caso de la distribución espacial, grupos de especies y clases de bosque. También se consideró la distribución de los incendios según las clases de tamaño, indicador que muestra la eficiencia del servicio de protección.

\section{RESULTADOS Y DISCUSIÓN}

Distribución de los incendios y sus afectaciones a través del periodo de años seleccionado. En la tabla 1 se muestra la distribución de los incendios y las afectaciones a través del periodo de años seleccionado. La frecuencia anual de incendios y la media de las afectaciones para Monte Alegre fueron de 72,75 y 40,34 ha respectivamente mientras que para Pinar del Río fueron de 91,0 y 3592,36 ha respectivamente. Se destacan los altos valores, en ambas variables, para el año 1999 en Pinar del Río. También en el caso de Monte Alegre en este año fue mayor el valor para la cantidad de incendios. 
Tabla 1: Ocurrencia de incendios y sus áreas quemadas en Monte Alegre y Pinar del Río 1998 - 2001

Table 1: Forest fire occurrence and burned area in Monte Alegre and Pinar del Río 1998-2001

\begin{tabular}{crrrrrrrr}
\hline \multirow{2}{*}{ Años } & \multicolumn{3}{c}{ Monte Alegre } & \multicolumn{6}{c}{ Pinar del Río } \\
\cline { 2 - 9 } & \multicolumn{2}{c}{ Incendios } & \multicolumn{2}{c}{ Afectaciones } & \multicolumn{2}{c}{ Incendios } & \multicolumn{1}{c}{ Afectaciones } \\
\cline { 2 - 9 } & $\mathbf{( N o )}$ & $\mathbf{( \% )}$ & $\mathbf{( h a )}$ & $\mathbf{( \% )}$ & $\mathbf{( N o . )}$ & $\mathbf{( \% )}$ & (ha) & $\mathbf{( \% )}$ \\
\hline 1998 & 22 & 7,56 & 5,75 & 3,56 & 85 & 23,35 & 1395,89 & 9,71 \\
1999 & 95 & 32,65 & 45,05 & 27,91 & 106 & 29,12 & 10975,80 & 76,38 \\
2000 & 90 & 30,93 & 55,67 & 34,49 & 79 & 21,70 & 936,99 & 6,52 \\
2001 & 84 & 28,87 & 54,92 & 34,03 & 94 & 25,82 & 1060,76 & 7,38 \\
\hline Totales & $\mathbf{2 9 1}$ & $\mathbf{1 0 0 , 0 0}$ & $\mathbf{1 6 1 , 3 9}$ & $\mathbf{1 0 0 , 0 0}$ & $\mathbf{3 6 4}$ & $\mathbf{1 0 0 , 0 0}$ & $\mathbf{1 4 3 6 9 , 4 4}$ & $\mathbf{1 0 0 , 0 0}$ \\
\hline
\end{tabular}

Distribución de los incendios y sus afectaciones a través del año.

Las figuras 1 y 2 muestran la distribución mensual de los incendios y las afectaciones. Como se observa no coinciden las épocas de incendios. Esto está muy relacionado con las variaciones climáticas a través del año en estos lugares situados en los hemisferios norte y sur. En el caso de Monte Alegre el 59,11\% de los incendios y el 81,31 $\%$ de las afectaciones ocurrieron durante el periodo agosto - octubre. En Pinar del Río el $41,49 \%$ de los incendios y el $85,96 \%$ de las afectaciones se presentaron los meses de abril y mayo.

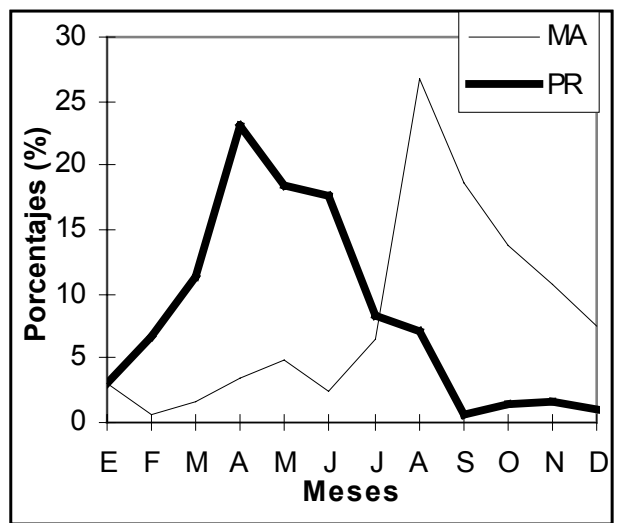

Figura 1: Distribución mensual de incendios en Monte Alegre (MA) y Pinar del Río (PR) 1998 - 2001 Figure 1: Fire distribution by month in Monte Alegre (MA) and Pinar del Rio (PR) 1998 - 2001

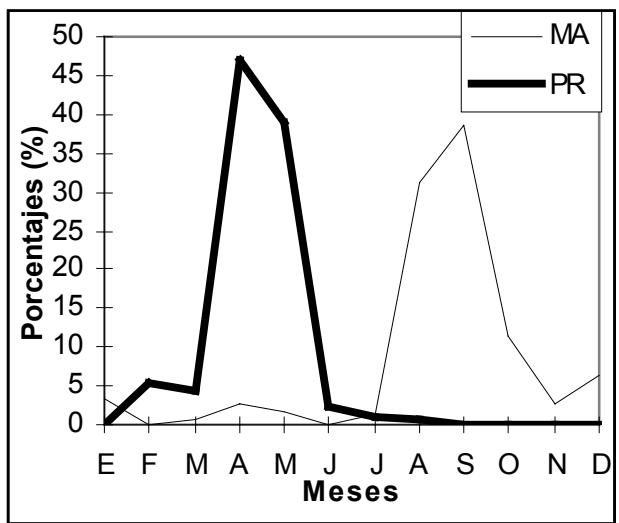

Figura 2: Distribución mensual de las afectaciones en Monte Alegre (MA) y Pinar del Río (PR) 1998 - 2001 Figure 2: Burned area distribution by month in Monte Alegre (MA) and Pinar del Río (PR) $1998-2001$ 
Distribución de los incendios y sus afectaciones a través del día.

En la figura 3 se observa la distribución diaria de los incendios. Entre las 14:00 y las 18:00 horas ocurrieron en Monte Alegre y Pinar del Río el 61,85 y el 73,36 \% de los incendios respectivamente. Esto se corresponde con las variaciones diarias de la temperatura y la humedad relativa, dos variables del tiempo atmosférico que influyen en la humedad de los materiales combustibles.
Distribución de los incendios a través de la semana.

En la figuras 4 se observa la distribución semanal de los incendios. No existe diferencia significativa para las ocurrencias entre los diferentes días de la semana. No obstante, los mayores valores se registraron los días laborables. Esto puede deberse a que durante estos días se incrementa el acceso de personas al bosque como consecuencia del desarrollo del proceso de producción forestal.

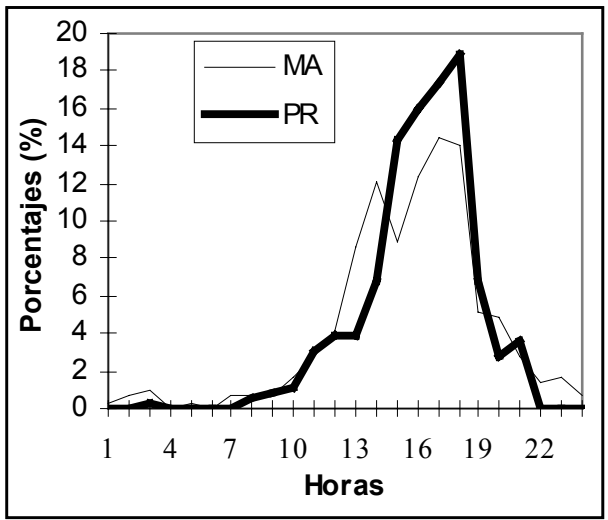

Figura 3: Distribución diaria de incendios en Monte Alegre (MA) y Pinar del Río (PR) 1998 - 2001

Figure 3: Forest fire occurrence by hours in Monte Alegre (MA) and Pinar del Río (PR) 1998 - 2001

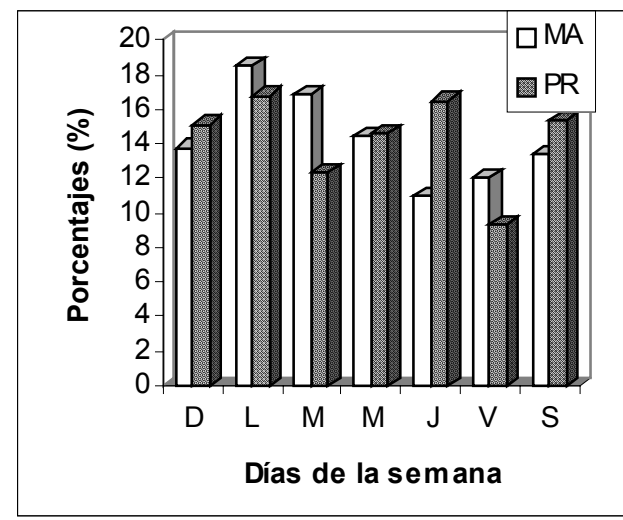

Figura 4: Distribución semanal de incendios en Monte Alegre (MA) y Pinar del Río (PR) 1998 - 2001

Figure 4: Fire distribution by week in Monte Alegre (MA) and Pinar del Río (PR) 1998 - 2001

Distribución de los incendios y sus afectaciones según los grupos de especies.

Según se observa en la tabla 2, durante el periodo del estudio en Monte Alegre la mayor cantidad tanto de incendios $(41,92 \%)$ como de hectáreas afectadas $(40,47 \%)$ se presentan en el grupo de otra vegetación. En Pinar del Río esto ocurrió en el grupo Pinus spp, correspondiendo a las ocurrencias el 67,03 $\%$ y a las afectaciones el $82,83 \%$ del total. 
Tabla 2: Ocurrencia de incendios y sus áreas quemadas por grupos de especies en Monte Alegre y Pinar del Río 1998 - 2001

Table 2: $\quad$ Forest fire occurrence and burned area by groups of species in Monte Alegre and Pinar del Río 1998 - 2001

\begin{tabular}{|c|c|c|c|c|c|c|c|c|}
\hline \multirow{3}{*}{$\begin{array}{l}\text { Grupos } \\
\text { de } \\
\text { Especies }\end{array}$} & \multicolumn{4}{|c|}{ Monte Alegre } & \multicolumn{4}{|c|}{ Pinar del Río } \\
\hline & \multicolumn{2}{|c|}{ Incendios } & \multicolumn{2}{|c|}{ Afectaciones } & \multicolumn{2}{|c|}{ Incendios } & \multicolumn{2}{|c|}{ Afectaciones } \\
\hline & (No.) & $(\%)$ & (ha) & $(\%)$ & (No.) & $(\%)$ & (ha) & $(\%)$ \\
\hline Pinus spp. & 92 & 31,62 & 42,87 & 26,56 & 244 & 67,03 & 11902,39 & 82,83 \\
\hline Eucalyptus spp. & 67 & 23,02 & 44,88 & 27,81 & 87 & 23,90 & 1556,00 & 10,83 \\
\hline Casuarina spp. & 1 & 0,34 & 0,04 & 0,02 & 3 & 0,82 & 11,80 & 0,08 \\
\hline Otras latifolias & 9 & 3,09 & 8,28 & 5,13 & 30 & 8,24 & 899,25 & 6,26 \\
\hline Otra vegetación & 122 & 41,92 & 65,32 & 40,47 & 0 & 0,00 & 0,00 & 0,00 \\
\hline Totales & 291 & 100,00 & 161,39 & $\mathbf{1 0 0 , 0 0}$ & 364 & 100,00 & 14369,44 & 100,00 \\
\hline
\end{tabular}

Distribución de los incendios y sus afectaciones según las clases de bosques.

En correspondencia con la tabla 3, la mayor cantidad de incendios y de sus afectaciones se presentan en los bosques artificiales, lo cuál es aún más notable para el caso de Pinar del Río. Esto puede deberse a su importante participación en los planes de producción y a que estos se ubican en áreas más accesibles a las personas.

Tabla 3: Ocurrencia de incendios y sus áreas quemadas por clases de bosques en Monte Alegre y Pinar del Río 1998 - 2001

Table 3: Forest fire occurrence and burned area by classes of forest in Monte Alegre and Pinar del Río 1998 2001

\begin{tabular}{|c|c|c|c|c|c|c|c|c|}
\hline \multirow{3}{*}{, } & \multicolumn{4}{|c|}{ Monte Alegre } & \multicolumn{4}{|c|}{ Pinar del Río } \\
\hline & \multicolumn{2}{|c|}{ Incendios } & \multicolumn{2}{|c|}{ Afectaciones } & \multicolumn{2}{|c|}{ Incendios } & \multicolumn{2}{|c|}{ Afectaciones } \\
\hline & (No.) & $(\%)$ & (ha) & $(\%)$ & (No.) & $(\%)$ & (ha) & $(\%)$ \\
\hline Naturales & 131 & 45,02 & 73,60 & 45,60 & 87 & 23,08 & 5296,11 & 36,86 \\
\hline Artificiales & 160 & 54,98 & 87,79 & 54,40 & 290 & 76,92 & 9073,33 & 63,14 \\
\hline Totales & 291 & 100,00 & 161,39 & 100,00 & 377 & 100,00 & 14369,44 & 100,00 \\
\hline
\end{tabular}

Distribución de los incendios y sus afectaciones según los grupos de causas.

La distribución de los incendios y sus afectaciones según los grupos de causas se muestra en la tabla 4. En Monte Alegre la principal causa de ocurrencias son los incendiarios los que provocan el 61,51\% del total, mientras que en Pinar del Río la causa más importante en este sentido son los rayos $(43,96 \%)$. Es interesante destacar que en ambos lugares los incendios originados por quema para limpieza son los que han provocado la mayor cantidad de las afectaciones.

Tabla 4: Ocurrencia de incendios y sus áreas quemadas por grupos de causas en Monte Alegre y Pinar del Río 1998 - 2001

Table 4: Forest fire occurrence and burned area by causes in Monte Alegre and Pinar del Río 1998 - 2001

\begin{tabular}{|c|c|c|c|c|c|c|c|c|}
\hline \multirow{3}{*}{ Causas } & \multicolumn{4}{|c|}{ Monte Alegre } & \multicolumn{4}{|c|}{$\begin{array}{l}\text { Pinar del Río } \\
\end{array}$} \\
\hline & \multicolumn{2}{|c|}{ Incendios } & \multicolumn{2}{|c|}{ Afectaciones } & \multicolumn{2}{|c|}{ Incendios } & \multicolumn{2}{|c|}{ Afectaciones } \\
\hline & (No.) & $(\%)$ & (ha) & $(\%)$ & (No.) & $(\%)$ & (ha) & $(\%)$ \\
\hline Rayos & 1 & 0,34 & 0 & 0,00 & 160 & 43,96 & 2128,07 & 14,81 \\
\hline Quema para Limpieza & 50 & 17,18 & 91,93 & 56,96 & 37 & 10,16 & 8691,60 & 60,49 \\
\hline Fumadores & 4 & 1,37 & 0,82 & 0,51 & 24 & 6,59 & 63,60 & 0,44 \\
\hline Incendiarios & 179 & 61,51 & 57,63 & 35,71 & 15 & 4,12 & 108,98 & 0,76 \\
\hline Estrada de Ferro & 3 & 1,03 & 1,6 & 0,99 & & & & \\
\hline Operaciones Forestales & 3 & 1,03 & 0,55 & 0,34 & 6 & 1,65 & 9,20 & 0,06 \\
\hline Diversos & 21 & 7,22 & 3,59 & 2,22 & 55 & 15,11 & 1531,80 & 10,66 \\
\hline Sub-total & 261 & & 156,12 & & 297 & & 12533,25 & \\
\hline No Determinadas & 30 & 10,31 & 5,27 & 3,27 & 67 & 18,41 & 1836,19 & 12,78 \\
\hline Total & 291 & 100,00 & 161,39 & 100,00 & 364 & 100,00 & 14369,44 & 100,00 \\
\hline
\end{tabular}


Distribución de los incendios y sus afectaciones por clases de tamaño.

La tabla 5 muestra la distribución de los incendios y sus afectaciones por clases de tamaño. En Monte Alegre el 66,32 \% de los incendios quedó en la clase I y el total en las clases I y II fue del 96,56 \%. En Pinar del Río solo el 4,40 \% quedó en la clase I y el 70,06 \% en las clases I y II. De acuerdo con esto, mientras que en Monte Alegre la gran mayoría de los incendios quema menos de 4,0 ha, en
Pinar del Río el 90,93 \% de los incendios queman entre 0,1 y 40,0 ha. Puede observarse que solo el 2,20\% de los incendios ubicados en la clase $\mathrm{V}$ provocaron el $85,83 \%$ de las afectaciones. Es evidente la necesidad de aumentar la eficiencia del servicio de protección contra incendios en Pinar del Río pues solo así será posible aumentar la concentración de los mismos en las clases de menor tamaño, reduciéndose de esta manera el total de área quemada.

Tabla 5: Ocurrencia de incendios y sus áreas quemadas por clases de tamaño en Monte Alegre y Pinar del Río $1998-2001$

Table 5: Forest fire occurrence and burned area by size classes in Monte Alegre and Pinar del Río 1998 2001

\begin{tabular}{|c|c|c|c|c|c|c|c|c|c|}
\hline \multirow{3}{*}{\multicolumn{2}{|c|}{$\begin{array}{c}\text { Clases } \\
\text { de } \\
\text { Tamaño }\end{array}$}} & \multicolumn{4}{|c|}{ Monte Alegre } & \multicolumn{4}{|c|}{ Pinar del Río } \\
\hline & & \multicolumn{2}{|c|}{ Incendios } & \multicolumn{2}{|c|}{ Afectaciones } & \multicolumn{2}{|c|}{ Incendios } & \multicolumn{2}{|c|}{ Afectaciones } \\
\hline & & (No.) & $(\%)$ & (ha) & $(\%)$ & (No.) & $(\%)$ & (ha) & (\%) \\
\hline I & $(0-0,09)$ & 193 & 66,32 & & 2,50 & 16 & 4,40 & 0,64 & 0,00 \\
\hline Il & $(0,1-$ & 88 & 30,2 & 76,16 & 47,19 & 239 & 65,66 & 392,00 & 2,73 \\
\hline II & $(4,1-40,0)$ & 10 & 3,44 & 81,20 & 50,31 & 92 & 25,27 & 976,60 & 6,80 \\
\hline & $(40,1-200,0)$ & 0 & 0,00 & 0,00 & 0,00 & 9 & 2,47 & 666,30 & 4,64 \\
\hline $\mathrm{V}$ & $(>200)$ & 0 & 0,00 & 0,00 & 0,00 & 8 & 2,20 & 12333,90 & 85,83 \\
\hline & Totales & 291 & 100,00 & 161,39 & 100,00 & 364 & 100,00 & 14369,44 & 100,00 \\
\hline
\end{tabular}

\section{CONCLUSIONES}

La existencia de datos sobre las ocurrencias de los incendios y sus afectaciones han permitido comparar dos regiones ubicadas en distintos hemisferios, con climas diferentes y con sistemas socioeconómicos también diferentes, Monte Alegre, Brasil y Pinar del Río, Cuba. No obstante, se ha podido establecer que existen similitudes y diferencias en cuanto a la manifestación del problema de los incendios forestales. Dentro de los resultados similares puede citarse que en 1999 en ambos lugares ocurrió la mayor cantidad de incendios. Sería interesante hacer este estudio durante un periodo mayor de años. También es muy similar la distribución diaria de los incendios, ocurriendo la gran mayoría entre las 14:00 y las 18:00 horas. Igualmente, los mayores valores se ocurrencias de incendios se registran los días laborables. Igualmente, la mayor cantidad de incendios y de sus afectaciones se presentan, en ambos lugares, en los bosques artificiales. Tanto en Monte Alegre como en Pinar del Río los incendios originados por quema para limpieza son los que han provocado la mayor cantidad de las afectaciones.

De acuerdo con el análisis son diferentes la época de incendios. La misma en Pinar del Río se presenta en los meses de abril y mayo y en Monte Alegre lo hace entre agosto y octubre. También son diferentes los grupos de vegetación más afectados por los incendios. En Monte Alegre los mayores valores corresponden al grupo de otra vegetación, y en Pinar del Río al grupo Pinus spp. En cuanto a las causas en Monte Alegre la principal para las ocurrencias son los incendiarios y en Pinar del Río la causa más importante en este sentido son los rayos. Otro indicador que mostró resultados diferentes fue el correspondiente a las clases de tamaño. En Pinar del Río, contrario a lo que sucedió para Monte Alegre, se ubican en la clase I una cantidad insignificante de los incendios. También puede mencionarse que en Monte Alegre la gran mayoría de los incendios quema menos de 4,0 ha, en Pinar del Río el 90,93\% de los mismos queman entre 0,1 y 40,0 ha. 


\section{REFERENCIAS}

Calabri, G. (1991) Problemas y perspectivas relativas a los incendios forestales, su prevención y su dominio. Actas del $10^{\circ}$ Congreso Forestal Mundial. París.

Çanakçioglu, H. (1990) Forest Fire in Turkey. Forest Fire Research and Protection from Fire (Proceedings) XIX IUFRO World Congress. Canada. 26 - 41 pp.

Ramos, M.P. 1999: Bases metodológicas para el perfeccionamiento de la prevención de los incendios forestales. Tesis presentada en opción al Grado Científico de Doctor en Ciencias Forestales. 140 p.

Ramos, M.P. 2002: Sistema Integrado para el Manejo de Bases de Datos sobre Incendios Forestales (SIMBDIF) Versión 1.2. Trabajo presentado en el III Congreso Forestal Venezolano.
Soares, R.V. (1988) Perfil dos incêndios florestais no Brasil, de 1984 a 1987. Revista Floresta. Brasil.

Soares, R.V. 1992: Ocurrência de incêndios florestais em reflorestamentos. I Seminario Nacional sobre Incêndios Florestais e Queimadas. Brasil, 15 p.

Soares, R.V. y G. Paez. 1974: Uma nova fórmula para determinar o grau de peligro de incendios florestais na região Centro Paranense. Revista Floresta. Trabajo presentado en el II Congreso Forestal de Brasil.

Vélez, R. (1990) Los incendios forestales en el Mediterráneo: perspectiva regional. Revista Unasylva. Vol.41, No. 162.3 - 9 pp.

Wadsworth, F.H. 2000: Producción Forestal para América Tropical. USDA. Servicio Forstal. Manual de Agricultura 710-s. 603 p. 